\title{
Immune-Mediated Hematological Disorders as Uncommon Presentation Forms of Systemic Lupus Erythematosus
}

\author{
Lucilene S R Resende ${ }^{1 *}$, Fabiana G Faustino ${ }^{1}$, Marilia R Resende ${ }^{2}$, Ligia Niero-Melo ${ }^{1}$ and Patricia C Garcia ${ }^{3}$ \\ ${ }^{1}$ Hematology Service of Internal Medicine Department, Botucatu Medical School, São Paulo State University, Brazil \\ ${ }^{2}$ Undergraduate Student of the Medical School, Minas Gerais Federal University, Brazil \\ ${ }^{3}$ Immunohematology Laboratory of the Botucatu Medical School, São Paulo State University, Brazil
}

Submission: March 25, 2021; Published: April 05, 2021

*Corresponding author: Lucilene S R Resende, Hematology Service of Internal Medicine Department, Botucatu Medical School, Sao Paulo State University, Brazil

Abstract

Background: It is well known that immune-mediated hematological disorders can precede, be concurrent with or follow a systemic lupus erythematosus (SLE) diagnosis. Some are relatively frequent and even consist in diagnosis criteria for this rheumatic disease. However, others are uncommonly seen as a presentation form of an SLE.

Objectives: To describe immune-mediated hematological disorders which are uncommonly presentation forms of systemic lupus erythematosus (SLE).

Material \& Methods: A retrospective study was carried out of patients showing immune-mediated hematological disorders uncommonly described as a presentation form of SLE.

Results: Six hematology patients were studied: four diagnosed with Evans syndrome (ES), one with thrombotic thrombocytopenic purpura (TTP), and one with adult-onset macrophage activation syndrome (MAS) as presentation forms of SLE. Two ES cases, the TTP and MAS cases were diagnosed concurrently with SLE. The other two ES cases preceded SLE by months or years. Some clinical, laboratory, and pathological features of these hematological disorders are discussed along with aspects of their association to SLE.

Discussion: Besides existing relatively frequent immune-mediated hematological disorders which even consist in diagnosis criteria for SLE, other hematological diagnoses can also uncommonly be seen as a presentation form of this rheumatic disease.

Conclusion: This paper reports three immune-mediated hematological disorders uncommonly seen at SLE presentation: ES, TTP, and adultonset MAS.

Keywords: Systemic Lupus Erythematosus; Evans Syndrome; Autoimmune Hemolytic Anemia; Immune Thrombocytopenic Purpura; Thrombotic Thrombocytopenic Purpura; Macrophage Activation Syndrome

Abbreviations: SLE: Systemic Lupus Erythematosus; ES: Evans Syndrome; TTP: Thrombotic Thrombocytopenic Purpura; MAS: Macrophage Activation Syndrome; AIHA: Autoimmune Hemolytic Anemia; ITP: Immune Thrombocytopenic Purpura; DAT: Direct Antiglobulin Test; LDH: Lactate Dehydrogenase; sHLH: Secondary Hemophagocytic Lymphohistiocytosis; MAS: Macrophage Activation Syndrome; IAT: Indirect Antiglobulin Test

\section{Introduction}

Systemic lupus erythematosus (SLE) is a worldwide heterogeneous autoimmune disorder that can affect many organs and tissues [1,2] due to interactions between genetic susceptibility, environmental triggers, and hormonal and immunological factors [2]. SLE diagnosis combines clinical and immunological findings
[3] that are usually heterogeneous in patients but can change over time in individual cases [1,2]. Hematologically, there are four clinical (hemolytic anemia, thrombocytopenia, leucopenia and lymphopenia in the absence of other known causes) and one immunological (positive direct antiglobulin test - DAT in the absence of hemolytic anemia) possible diagnostic criteria 
for SLE, being that one or more may be present at SLE diagnosis [3]. However, a given hematologic disorder affecting one or more blood cell lineages can emerge at any moment throughout the course of SLE [4-6], and can even be the initial manifestation, preceding other manifestations by months or even years [7]. Although autoimmune cytopenias are most typically published, other hematological findings which are not consistent with SLE diagnosis criteria have rarely been reported in association to this rheumatic disease $[4,6-13]$. The goal of this study is to present cases of patients admitted by hematologists with initial immunemediated hematological disorders that are uncommonly seen as a first manifestation of SLE, which was then subsequently diagnosed at varying time spans after admission.

\section{Methods}

A retrospective study was performed on patients admitted to the hematology ward of Botucatu Medical School, São Paulo State University, Botucatu, SP, Brazil, between January 2006 and December 2015. Patients were over 18 years old and had been admitted by hematologists with initial immune-mediated hematological disorders uncommonly associated with SLE which was subsequently diagnosed by them meeting the required classification criteria of the Systemic Lupus International Collaborating Clinics (2012 SLICC) [3], over varying time spans from first hematological assistance. Immune-mediated hematological disorders uncommonly associated with SLE were considered as those other than isolated autoimmune hemolytic anemia (AIHA), immune thrombocytopenic purpura (ITP), leucopenia or lymphopenia which are most commonly described in SLE patients. The concepts used in this study for AIHA, immune thrombocytopenia and immune leucopenia/lymphopenia were those adopted by the 2012 SLICC Classification Criteria [3]: hemolytic anemia with raised reticulocytes and positive direct antiglobulin test (DAT), isolated platelet count below $100 \times 10^{3} /$ $\mathrm{mm}^{3}$, and leucocyte count below $4 \times 10^{3} / \mathrm{mm}^{3}$ or lymphocyte count below $1 \times 10^{3} / \mathrm{mm}^{3}$ separately. Other causes for thrombocytopenia, leucopenia or lymphopenia might be absent. Anemia was defined as hemoglobin less than $12 \mathrm{~g} / \mathrm{dL}$ in women and $13.5 \mathrm{~g} / \mathrm{dL}$ in men [14].

The serum biochemistry criteria definer of hemolysis included raised lactate dehydrogenase (LDH) and unconjugated bilirubin, and low haptoglobin levels. Evans syndrome (ES) was diagnosed by the concurrent or sequential occurrence of AIHA and ITP [7]. Recurrent ES meant a reappearance of both clinical and laboratory findings of this autoimmune disorder after any asymptomatic period. Thrombotic thrombocytopenic purpura (TTP) was defined as a thrombotic microangiopathy characterized by microangiopathic hemolytic anemia, severe thrombocytopenia, organ ischemia linked to disseminated microvascular platelet rich-thrombi, and functional deficiency of ADAMTS-13 [15]. Macrophage activation syndrome (MAS) was defined as a secondary hemophagocytic lymphohistiocytosis (sHLH) associated with rheumatic diseases being characterized by clinical and laboratory features such as sustained fever, pancytopenia, liver dysfunction, hyperferritinemia, and fibrinolytic coagulopathy [12]. Clinical and laboratory findings related to immune-mediated hematologic disorders and SLE were obtained from patient medical records, and included: gender, ethnicity, age, blood cell counts, DAT, indirect antiglobulin test (IAT), DAT and/or IAT antibody specificity, time span between hematological and SLE diagnosis, hematological treatment during hospitalization, and outcome. Pictures were taken from patient electronic image files and from the hematology archive of bone marrow slides. This study was approved by the Human Research Ethics Committee of the Institution.

Results

Table 1: Clinical and laboratory characterization of 4 patients diagnosed with ES before SLE.

\begin{tabular}{|c|c|c|c|c|c|c|c|c|}
\hline Case & Gender & Ethnicity & $\begin{array}{c}\text { Age } \\
\text { (years) }\end{array}$ & $\begin{array}{l}\mathrm{Hb}(\mathrm{g} / \mathrm{dL}) / \text { Ret. } \\
\text { (\%) / Platelet } \\
\text { count }\end{array}$ & $\begin{array}{c}\text { DAT / } \\
\text { Antibody } \\
\text { specificity }\end{array}$ & $\begin{array}{c}\text { IAT/ } \\
\text { Antibody } \\
\text { specificity }\end{array}$ & $\begin{array}{l}\text { Time span } \\
\text { between ES and } \\
\text { SLE diagnosis }\end{array}$ & $\begin{array}{l}\text { Hematological } \\
\text { treatment / outcome }\end{array}$ \\
\hline 1 & Female & Caucasian & 29 & $\begin{array}{c}5.0 / 7.6 / \\
54.000\end{array}$ & $\operatorname{IgG}+\mathrm{C} 3 \mathrm{~d} / \mathrm{NS}$ & $\begin{array}{c}+/ \text { anti-E, } \\
\text { anti-K, anti-Jk }\end{array}$ & $\leq 7$ days $*$ & $\begin{array}{c}\text { corticosteroids + IVIG } \\
+ \text { RBC / obit }\end{array}$ \\
\hline 2 & Female & Caucasian & 15 & $\begin{array}{c}5.6 / 3.9 / \\
1.000\end{array}$ & $\operatorname{IgG}+\mathrm{C} 3 \mathrm{~d} / \mathrm{NS}$ & $+/ \mathrm{NS}$ & $\leq 7$ days $* *$ & $\begin{array}{l}\text { corticosteroids + IGIV } \\
\text { + RBC / discharge }\end{array}$ \\
\hline 3 & Female & Caucasian & 29 & $\begin{array}{c}4.8 / 5.0 / \\
10.000\end{array}$ & IgG / NS & $\begin{array}{l}+ \text { / anti-C, } \\
\text { anti-K }\end{array}$ & 8 months & $\begin{array}{c}\text { corticosteroids + IGIV } \\
+ \text { RBC / discharge }\end{array}$ \\
\hline 4 & Female & Caucasian & 50 & $\begin{array}{c}5.1 / 5.5 / \\
63.000\end{array}$ & $\operatorname{IgG}+\mathrm{C} 3 \mathrm{~d} / \mathrm{NS}$ & $+/ \mathrm{NS}$ & $43^{* * *}$ months & $\begin{array}{l}\text { corticosteroids }+ \\
\text { IVIG }+ \text { RBC + platelet } \\
\text { apheresis / discharge }\end{array}$ \\
\hline
\end{tabular}

Hb: hemoglobin; Ret.: reticulocytes; DAT: direct antiglobulin test; Ig: immunoglobulin; C: complement; NS: non-specific; IAT: indirect antiglobulin test; *: previously diagnosed glomerulonephritis; **: previous recurrent thrombocytopenia; ***: recurrent ES symptoms; IVIG: intravenous immunoglobulin; RBC: red blood cell 
We studied 6 patients with initial hematological diagnoses: 4 of ES, 1 of TTP, and 1 of MAS. Table 1 shows data from the 4 patients initially diagnosed with ES (Table 1). They were Caucasian female with an average age of 30 years. At diagnosis average hemoglobin, reticulocyte count and platelet count were $5.1 \mathrm{~g} / \mathrm{dL}, 5.5 \%$ and $32 \times 10^{3} / \mathrm{mm}^{3}$ respectively. There were no cases with leucopenia or lymphopenia. A positive DAT revealed IgG + C3d in 3 patients, and only IgG in one. Autoantibodies showed no specificity for any erythrocyte-antigen in all patients. IAT were also positive in the 4 patients, with only 2 showing defined specificity: one had serum anti-E, anti-K, and anti-Jka, and the other anti-C and anti-K. SLE diagnosis was performed $\leq 7$ days after admission in 2 patients, one having been referred with glomerulonephritis diagnosed by kidney biopsy 14 years earlier, and another had reported asymptomatic episodes of thrombocytopenia over the last 4 years. In the remaining 2 patients SLE was diagnosed 8 and 43 months, respectively, after first ES episode with the latter showing several hematological relapses between both diagnoses. The 2012 SLICC criteria for SLE diagnosis found in these 4 patients were: hematological abnormalities (4), immunological abnormalities (4), joint symptoms (3), renal abnormalities (3), and oral ulcers (1). The hematological treatment for ES consisted of corticosteroids (4), IVIG (4), and RBC transfusion (4). One patient had major gastrointestinal bleeding due to a peptic ulcer and was also submitted to platelet apheresis transfusion.

A 22-year-old Caucasian female was referred to the university hospital displaying fever, paleness, mild mental confusion, and the symptoms/signs of left parietal syndrome. She reported several episodes of uninvestigated macroscopic hematuria in the last 5 years. Her hemoglobin, reticulocyte count and platelet count were $8.3 \mathrm{~g} / \mathrm{dL}, 4.4 \%$ and $16 \times 10^{3} / \mathrm{mm}^{3}$ respectively. There was no leucopenia but she did have a lymphopenia of $0.6 \times 10^{3} / \mathrm{mm}^{3}$. Numerous schistocytes were seen on the stained peripheral blood smear, and DAT plus IAT were both negative. Serum LDH was $830 \mathrm{U} / \mathrm{L}$, unconjugated bilirubin was $2.3 \mathrm{mg} / \mathrm{dL}$, haptoglobin was undetectable, and ADAMTS13 activity was $<10 \%$. Brain CT scan and MRI showed an extensive hemorrhagic lesion compromising the left parietal lobe with adjacent edema (Figures 1A \& 1B). A small hemorrhagic lesion posteriorly in the right parietal lobe was also seen in CT scan (Figure 1A). TTP was promptly diagnosed at admission, and SLE diagnosis was performed within 7 days according to 2012 SLICC criteria revealing: cutaneous, renal, neurologic, and immunological abnormalities. The hematological treatment for TTP consisted of plasmapheresis, corticosteroids, rituximab, and RBC plus platelet apheresis transfusions.

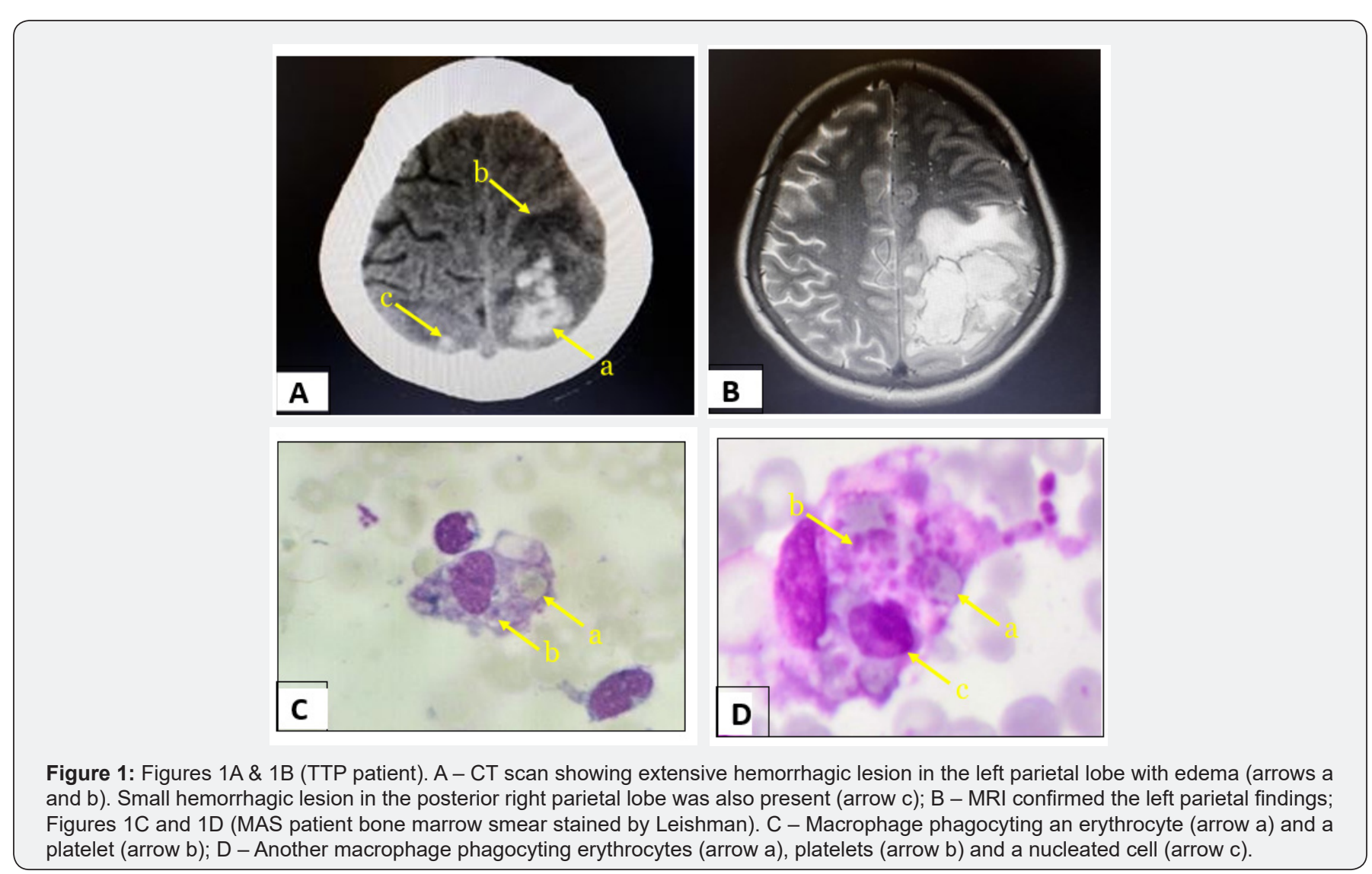


Another Caucasian female, 30 years old, was admitted with fever, adenopathy and mild splenomegaly without any identified infection or neoplasia. No clinical or laboratory findings of hemolysis were found. Her hemoglobin, reticulocyte and platelet counts were $8.1 \mathrm{~g} / \mathrm{dL}, 0.74 \%$ and $26 \times 10^{3} / \mathrm{mm}^{3}$ respectively. There was a mild leucopenia of $3.3 \times 10^{3} / \mathrm{mm}^{3}$ plus lymphopenia of 0.8 $\mathrm{x} 10^{3} / \mathrm{mm}^{3}$. A monocyte was seen phagocyting erythrocytes on stained peripheral blood smear. She showed an AST of $88 \mathrm{U} / \mathrm{L}$, ALT of $50 \mathrm{U} / \mathrm{L}$, serum ferritin $>1.650 \mathrm{ng} / \mathrm{mL}$, fibrinogen of $130 \mathrm{mg} /$ $\mathrm{dL}$, and International Normalized Ratio (INR) of 1.5. DAT and IAT were both negative. A stained bone marrow smear showed histiocytes in active hemophagocytosis of erythrocytes, platelets and nucleated cells (Figures 1C \& 1D). A diagnosis of MAS was established with a further diagnosis of SLE performed $\leq 7$ days later. After clinical history evaluation and laboratory tests, the 2012 SLICC criteria for SLE diagnosis observed in this patient were: hematologic abnormality (lymphopenia was considered), oral ulcers, cutaneous abnormality, joint symptoms, serositis, renal abnormality, neurologic abnormality and immunologic abnormalities. The hematological treatment for MAS consisted of intravenous corticosteroids and etoposide, as well as a RBC transfusion. All patients were discharged from the hematology ward, except for the one diagnosed with ES and SLE who died after a severe gastrointestinal bleeding whilst still in hospital.

\section{Discussion}

All studied patients were female with 5 being of reproductive age in accordance with SLE literature [2]. As soon as the immune-mediated hematological diagnoses were established, the hematologists promptly proceeded to investigate underlying diseases as this set of hematological disorders may occur secondarily to both underlying non-immunological and immunological conditions, including SLE [10]. Four of our 6 studied patients had a diagnosis of ES which corresponds to a rare hematological disorder representing up to $7 \%$ of AIHA and $2 \%$ of ITP [16]. Studies have shown that $27 \%$ to $50 \%$ of adult onset ES are usually secondary to another disorder, particularly hematological malignancies or SLE [16]. Zhang et al. (2016) reported that 3\% to $15 \%$ of ES patients develop SLE during the follow-up [7]. On the other hand, Jaime-Perez et al. (2018) reported that $1.7 \%$ to $2.7 \%$ of SLE patients showed secondary ES [18]. A recently published multicenter multiethnic cohort study did not find ES as a manifestation of early SLE in 389 studied patients [17]. In fact, they reported only isolated thrombocytopenia in $6.6 \%$ and AIHA in $4.6 \%$ of their patients without mentioning both together in any single SLE patient [18]. Regarding AIHA, all ES patients had severe warm-type hemolysis showing membrane-bound IgG autoantibodies, with 3 also having C3d. The autoimmune etiology for a concerning hemolytic anemia is demonstrated by a positive DAT [19] in 95\% of cases [20].

A positive DAT usually demonstrates warm AIHA with a combination of IgG and complement (usually C3d) bound to the red cell membrane; Leger \& Borge Jr et al. (2017) reported that warm-type AIHA uses to have both IgG with complement in $67 \%$ of cases, IgG without complement in $20 \%$, and complement without IgG in 13\% [21]. Studies have shown that ES-related AIHA is usually caused by IgG and rarely IgA, although cold agglutinins have not been found [16]. About $50 \%$ of all AIHA, mainly warm-type, are secondary to other diseases or medications [20]. These diseases comprise of immunodeficiency disorders, lymphoproliferative diseases, solid tumors, some viral infections, and rheumatic conditions [20,22], including SLE [6,19,23,24]. The main mechanism for hemolysis in these cases is by autoantibodies with or without complement involvement [22], as observed in this study. However, several cellular immune effectors have increasingly been recognized as participating in the hemolytic process, along with cytokine dysregulation and ineffective bone marrow compensation [22]. In our study, the ES autoantibodies obtained from erythrocyte surfaces by elution lacked specificity in all 4 cases, meaning that the antibodies reacted in vitro against the whole panel of phenotyped erythrocytes, in accordance with AIHA literature [20,21]. IAT were also positive in all studied ES patients. They were nonspecific in 2, also meaning they reacted in vitro against the whole panel of phenotyped erythrocytes, probably as result of copious serum free autoantibodies coming from the erythrocyte membranes, in accordance with reports in AIHA literature [25]. However, serum antibodies were identified from their positive IAT in 2 patients, which usually happen for alloantibodies [20]. These alloantibodies were directed against high immunogenic systems such as Rh (antigens C and E), Kell (antigen $\mathrm{K}$ ) and Kidd (antigen Jka). Immunization to red cell antigens may result from pregnancy, transfusion, transplantation, needle sharing, or injections of immunogenic material [26]. One patient in this study was a multiparous and another had received an RBC transfusion related to a previous complicated surgery, when they were probably sensitized. With respect to ITP, 2 ES patients displayed severe thrombocytopenia which placed them as at risk of spontaneous life-threatening bleeding $[27,28]$. Another 2 showed platelet counts $\geq 50 \times 10^{3} / \mathrm{mm}^{3}$ which usually provide safe hemostatic conditions unless there are other simultaneous predisposing injuries. As in AIHA, ITP may be secondary to underlying disorders, including SLE or other morbid conditions [27]. Regarding secondary ITP, as observed in this study, failures might occur in central B-lymphocyte tolerance checkpoints that operate during primary B-cell maturation in bone marrow leading to the formation of autoreactive antibodies [29]. These autoantibodies then cause an autoimmune IgG attack on platelets and megakariocytes mainly targeting their surface glycoproteins GPIIb-IIIA and GPIb-IX-V [30]. Antibody bound platelets are prematurely destroyed by the reticuloendothelial system in addiction to megakaryocyte function inhibition $[27,30]$. However, immunological mechanisms other than autoantibodies also seem to be involved in ITP pathogenesis including abnormalities in T helper cells, Tregs cells and CD8 lymphocytes [27]. After hematological treatment, 3 patients had their dual autoimmune 
conditions stabilized. Although secondary ES seems to have increased mortality compared to primary ES [16], only 1 studied patient died after a fatal bleeding whilst still on the hematological ward.

The fifth patient was a young female who presented a typical microangiopathic hemolytic anemia [15] with low ADAMTS13 activity whose diagnosis was acquired TTP. Such a rare disease results from autoantibodies against ADAMTS13, a von Willebrandcleaving protease, leading to formation of ultra-large von Willebrand factor multimers with unregulated platelet adhesion and aggregation, disseminated microthrombi, organ ischemia, and high erythrocyte shearing rates $[9,15,31,32]$. SLE was diagnosed sequentially. It is known that acquired TTP can be secondary to other disorders including autoimmune diseases, notably SLE [31]. Actually, SLE can be diagnosed before or concurrently with TTP diagnosis, or even following its recovery [33]. This patient showed neurological signs and symptoms which images studies revealed an extensive hemorrhagic lesion in the left parietal lobe and a small hemorrhage in the posterior right parietal lobe. In fact, the classic TTP diagnostic pentad includes neurological findings [32]. Although unusual, TTP can cause ischemic strokes as result of platelet thrombi in the brain vessels of young patients [34]. Sequentially, a TTP-related thrombocytopenia might bring about a secondary hemorrhage. Therefore, TTP itself could have caused the patient's brain lesions. However, SLE may also have triggered a brain vessel disease [35], predisposing her brain to secondary hemorrhage by TTP-induced thrombocytopenia. In such circumstances, TTP and SLE could have contributed to this patient's brain stroke. After hematological treatment she experienced clinical and laboratory improvement and was referred to the rheumatology service.

The final studied patient was admitted to hematology due to a suspected hematological neoplasia as she was presenting fever, adenopathy, splenomegaly and pancytopenia. It is well known that this set of clinical findings are common to many malign or benign disorders in medicine. Soon after admission monocyte phagocyting erythrocytes were seen on her stained peripheral blood smear. Given that she was pancytopenic a bone marrow aspiration was performed which revealed a notorious pan-hemophagocytosis. Concomitant serum biochemistry tests, mainly supported by extreme hyperferritinemia, pointed to a diagnosis of MAS and to a search for an underlying disease. Thus, SLE was promptly found. Macrophage activation syndrome is rare, mainly in adults, and frequently underrecognized $[13,36]$. It is well-known in pediatric and adolescent rheumatology, although it has increasingly been recognized in adult-onset population as a consequence of malignancy, infection, inflammation, autoimmunity, or a combination of these factors [36]. Most cases of MAS in adults are associated with Still's disease [13].

MAS is described during most rheumatic diseases and has been reported in up to $9 \%$ of SLE cases [36] but is uncommon as its presentation form [13]. It is caused by an inappropriate function of cytotoxic T lymphocytes and NK cells for clearing antigens. Dysfunctional immunological cells produce proinflammatory cytokines such as interferon $\gamma$ and tumor necrosis factor $\alpha$ that activate and recruit macrophages to tissues $[12,36,37]$. Dysfunction of the innate immune system also seems to be involved [36]. The phenomenon perpetuates immune stimulation creating a hyperinflammatory state called cytokine storm [36,37] which leads to high fever and tissue infiltration by activated macrophages and lymphocytes, provoking lifethreatening multi-organ inflammation and damage [37]. MAS is not exactly an autoimmune disorder in which autoantibodies are involved as occurring in those 5 patients described before. It seems more appropriate to designate MAS, generically, as an immune dysregulation. After initial hematological assistance and treatment, the patient was stabilized and then referred to the rheumatology service. This paper discusses rare immunemediated hematological disorders uncommonly seen as presentation form of SLE, suggesting that this underlying disease should always be considered by physicians when any evidence of autoimmunity or another kind of immune dysfunction involves the hematological system. Moreover, it reinforces the fact that SLE may be diagnosed too late after initial immune-mediated hematological disorder during patient follow-up.

\section{Conflicts of Interest}

The authors report no conflicts of interest.

\section{References}

1. Kuhn A, Bonsmann G, Anders H, Herzer P, Tenbrock K, Schneider M (2015) The Diagnosis and Treatment of Systemic Lupus Erythematosus. Dtsch Arztebl Int 112(25): 423-432.

2. Fava A, Petri M (2019) Systemic Lupus Erythematosus: Diagnosis and Clinical Management. J Autoimmun 96: 1-30.

3. Petri M, Orbai A, Alarcón GS, Gordon C, Merril JT, Fortin PR et al. (2012) Derivation and Validation of Systemic Lupus International Collaborating Clinics Classification Criteria for Systemic Lupus Erythematosus. Arthritis Rheum 64(8): 2677-2686.

4. Newman K, Owlia MB, El-Hemaidi I, Akhtari M (2013) Management of immune cytopenias in patients with systemic lupus erythematosus Old and new. Autoimmunity Reviews 12(7): 784-791.

5. Aleem A, Alarfaj AS, Khalil N, Alarfaj H (2014) Haematological abnormalities in systemic lupus erythematosus. Acta Reumatol Port 39: 236-241.

6. Fayyaz A, Igoe A, Kurien BT, et al. (2015) Haematological manifestations of lupus. Lupus Science \& Medicine 2: e000078.

7. Zhang L, Wu X, Wang L, Li J, Chen H, Zhao Y, et al. (2016) Clinical Features of Systemic Lupus Erythematosus Patients Complicated With Evans Syndrome. A Case-Control, Single Center Study. Medicine (Baltimore) 95(15): e3279.

8. Pereira RM, Velloso ER, Menezes Y, Gualandro S, Vassalo J, Yoshinari NH (1998) Bone marrow findings in systemic lupus erythematosus patients with peripheral cytopenias. Clin Rheumatol 17(3): 219-222. 
9. Abu-Hishmeh M, Sattar A, Zarlasht F, Ramadan M, Abdel-Rahman A, Hinson S, et al. (2016) Systemic Lupus Erythematosus Presenting as Refractory Thrombotic Thrombocytopenic Purpura: A Diagnostic and Management Challenge. A Case Report and Concise Review of the Literature. Am J Case Rep 17: 782-787.

10. Velo-García A, Castro SG, Isenberg DA (2016) The diagnosis and management of the haematologic manifestations of lupus. Journal of Autoimmunity 74: 139-160.

11. Chalayer E, Costedoat-Chalumeau N, Beyne-Rauzy O, Ninet J, Durupt S, Tebib J, et al. (2017) Bone marrow involvement in systemic lupus erythematosus. QJM: An International Journal of Medicine 110(11): 701-711.

12. . Crayne CB, Albeituni S, Nichols KE, Cron RQ (2019) The Immunology of Macrophage Activation Syndrome. Front Immunol 10: 119.

13. Gilboa M, Bornstein G, Ben-Zvi I, Grossman C (2020) Macrophage activation syndrome complicating rheumatic diseases in adults: casebased review. Rheumatol Int 40(4): 663-669.

14. WHO - World Health Organization (2011) Haemoglobin Concentrations for the Diagnosis of Anaemia and Assessment of Severity, Vitamin and Mineral Nutrition Information System. Geneva, Switzerland.

15. .Joly BS, Coppo P, Veyradie A (2017) Thrombotic thrombocytopenic purpura. Blood 129(21): 2836-2846

16. Audia S, Grienay N, Mounier M, Michel M, Bonnotte B (2020) Evans' Syndrome: From Diagnosis to Treatment. J Clin Med 9(12): 3851-3872.

17. Jaime-Pérez JC, Aguilar-Calderón PE, Salazar-Cavazos L, GómezAlmaguer D (2018) Evans syndrome: clinical perspectives, biological insights and treatment modalities. J Blood Med 9: 171-184.

18. Mosca M, Costenbader KH, Johnson SR, Lorenzoni V, Sebastiani GD, Hoye BF, et al. (2019) How Do Patients with Newly Diagnosed Systemic Lupus Erythematosus Present? A Multicenter Cohort of Early Systemic Lupus Erythematosus to Inform the Development of New Classification Criteria. Arthritis Rheumatol 71(1): 91-98.

19. Hill A, Hill QA (2018) Autoimmune hemolytic anemia. Hematology Am Soc Hematol Educ Program 30(1): 382-389.

20. Brodsky RA (2019) Warm Autoimmune Hemolytic Anemia. N Engl J Med 381: 647-654.

21. Leger RM, Borge Jr PD (2017) The Positive Direct Antiglobulin Test and Immune-Mediated Hemolysis. In: Fung MK, Eder AF, Spitalnik SL, Westhoff CM. Technical Manual. 19 ${ }^{\text {th }}$ ed. Bethesda: AABB pp. 385-404.

22. Barcellini W, Zaninoni A, Giannotta JA, Fattizzo B (2020) New Insights in Autoimmune Hemolytic Anemia: From Pathogenesis to Therapy. J Clin Med 9(12): 3859-3877.

23. Gormezano NWS, Kern D, Pereira OL, Esteves GCX, Sallum AME, Aikawa NE, et al.(2017) Autoimmune hemolytic anemia in systemic lupus erythematosus at diagnosis: differences between pediatric and adult patients. Lupus 26(4): 426-430.

24. Liebman HA, Weitz, IC (2017) Autoimmune Hemolytic Anemia. Med Clin N Am 101: 351-359.

25. Harm SK, Dunbar NM (2017) Transfusion-Service-Related Activities: Pretransfusion Testing and Storage, Monitoring, Processing, Distribution, and Inventory Management of Blood Components. In: Fung MK, Eder AF, Spitalnik SL, Westhoff CM. Technical Manual. 19 ed. Bethesda AABB pp. 467-487.

26. Hamilton JR, Bailey DJ (2017) Identification of Antibodies to Red Cell Antigens. In: Fung MK, Eder AF, Spitalnik SL, Westhoff CM. Technical Manual. $19^{\text {th }}$ ed. Bethesda, AABB pp. 349-384.

27. Cooper N, Ghanima W (2019) Immune Thrombocytopenia. N Eng J Med 381(10):945-955.

28. Neunert C, Terrell DR, Arnold DM, Buchanan G, Cines DB, Cooper N, et al. (2019) American Society of Hematology 2019 guidelines for imune thrombocytopenia. Blood Advances 3(23): 3829-3866.

29. Cines DB, Bussel JB, Liebman HA, Prak ETL (2009) The ITP syndrome: pathogenic and clinical diversity. Blood 113(26): 6511-6521.

30.Witkowski M, Witkowska M, Robak T (2019) Autoimmune Thrombocytopenia: Current treatment options in adults with a focus on novel drugs. Eur J Haematol 103: 531-41.

31. Yue C, Su J, Fan X, Song L, Jiang W, Xia J, et al.(2020) Immune-mediated thrombotic thrombocytopenic purpura in patients with and without systemic lupus erythematosus: a retrospective study. Orphanet J Rare Dis 15(1): 225-233.

32. Sukumar S, Lämmle B, Cataland SR (2021) Thrombotic Thrombocytopenic Purpura: Pathophysiology, Diagnosis, and Management. J Clin Med 10(3): 536-559.

33. George NG (2018) TTP: long-term outcomes following recovery. Hematology Am Soc Hematol Educ Program 2018(1): 548-552.

34. Tomich C, Debruxelles S, Delmas Y, Sagnier S, Poli M, Olindo S, et al. (2018) Immune-Thrombotic Thrombocytopenic Purpura is a Rare Cause of Ischemic Stroke in Young Adults: Case Reports and Literature Review. J Stroke Cerebrovasc Dis 27(11): 3163-3171.

35. McGlasson S, Wiseman S, Wardlaw J, Dhaun N, Hunt DPJ (2018) Neurological Disease in Lupus: Toward a Personalized Medicine Approach. Frontiers Immunol 9: 1146.

36. Carter SJ, Tattersall RS, Ramanan AV (2019) Macrophage activation syndrome in adults: recent advances in pathophysiology, diagnosis and treatment. Rheumatol 58: 5-17.

37. Sen ES, Steward CG, Ramanan AV (2017) Diagnosing haemophagocytic syndrome. Arch Dis Child 102: 279-284. 


\section{Your next submission with Juniper Publishers} will reach you the below assets

- Quality Editorial service

- Swift Peer Review

- Reprints availability

- E-prints Service

- Manuscript Podcast for convenient understanding

- Global attainment for your research

- Manuscript accessibility in different formats ( Pdf, E-pub, Full Text, Audio)

- Unceasing customer service

Track the below URL for one-step submission https://juniperpublishers.com/online-submission.php 